\title{
SEMICONTINUITY OF KODAIRA DIMENSION
}

\author{
BY D. LIEBERMAN ${ }^{1}$ AND E. SERENESI
}

Communicated by Edgar Brown, Jr., January 10, 1975

Let $X$ be a compact analytic space (or a complete algebraic variety) and let $L$ be a line bundle on $X$ and denote by $f_{i}: X \rightarrow \mathbf{P}^{N}$ the rational map defined by the global sections of $L^{\otimes i}$. The $L$-dimension of $X, K(X, L)$ is defined by

$$
K(X, L)=\varlimsup_{i \rightarrow \infty}\left(\operatorname{dim}\left(f_{i}(X)\right)\right.
$$

with the convention $K(X, L)=-\infty$ if $L^{\otimes i}$ has no nontrivial sections for all $i>0$. In the particular case when $X$ is nonsingular and $L=\Omega$ is the canonical bundle, the invariant $K(X)=K(X, \Omega)$ is called the canonical (or Kodaira) dimension of $X$ and is the fundamental invariant in the classification of surfaces. Recent works by Ueno [4] and Iitaka [1], [2] have studied $K(X, L)$ for higher dimensional varieties. A fundamental open question is the behavior of $K(X, \mathrm{~L})$ under deformations of $(X, \mathrm{~L})$. When $X$ is a smooth surface the plurigenera (and hence the Kodaira dimension) are deformation invariant [1], and litaka has constructed a family of threefolds $X_{t}$ with $K\left(X_{0}\right)=0$ and $K\left(X_{t}\right)=-\infty, t \neq 0$.

Our main result is

THEOREM. Given $X_{0}$ a compact analytic space (or complete algebraic variety) and $L_{0}$ a line bundle on $X_{0}$ satisfying

(1) $L_{0}^{\otimes_{i}}$ is spanned by its global sections for some $i>0$,

(2) $K\left(X_{0}, L_{0}\right)=\operatorname{dim}\left(X_{0}\right)$, and $\left(X_{t}, L_{t}\right)$ is any (flat) deformation of $\left(X_{0}, L_{0}\right)$, then $K\left(X_{t}, L_{t}\right)=$ $K\left(X_{0}, L_{0}\right)$.

When $X_{0}$ is a smooth surface and $L_{0}=\Omega_{0}$ it was shown by Mumford [3] that hypothesis (1) on $L_{0}$ is implied by (2). For general $L_{0}$ hypothesis

AMS (MOS) subject classifications (1970). Primary 14D15, $32 \mathrm{G} 05$.

Key words and phrases. Kodaira dimension, semicontinuity, deformation.

1 Sloan Foundation Fellow, partially supported by NSF GP-28323A3. 
(1) is not implied by (2); however when $X_{0}$ is smooth and $L_{0}=\Omega_{0}$ the first hypothesis might be unnecessary.

The general line of argument is the following:

Given $\pi: X \rightarrow S$ proper and flat and $L \longrightarrow X$ a line bundle one defines a relative $L$-dimension $K(X \mid S, L)$ as follows. Let $f_{i}: X \rightarrow \operatorname{Proj}\left(\pi_{*} L^{\otimes i}\right)$ be the rational map defined by $\pi^{*}\left(\pi_{*} L^{\otimes_{i}}\right) \rightarrow L^{\otimes_{i}}$. Let

$$
K(X \mid S, L)=\widetilde{\lim }\left(\operatorname{dim} f_{i}(X)\right)-\operatorname{dim}(S)
$$

(or $-\infty$ if $\pi_{*}\left(L^{\otimes}\right)=0$ for all $\left.i>0\right)$.

Proposition 1. $K\left(X_{s}, L_{s}\right) \geqslant K(X \mid S, L)$ for all $s \in S$ with equality for $s \in W$ a nonempty c-open subset of $S$ (i.e. $W$ is the complement of a countable union of subvarieties).

As an immediate corollary one sees that the L-dimension is upper semicontinuous in the topology defined by $c$-open sets. The set $W$ and its complement may both be dense, e.g. taking $X_{s} \stackrel{\sim}{\rightarrow} X_{0}$ a curve of genus $g>0, S$ the Jacobian of $X_{0}$ and $L_{s}$ the canonical family of degree zero bundles, one finds $W=S-$ \{points of finite order .

The main theorem follows from

Proposition 2. If $K\left(X_{s}, L_{s}\right)=\operatorname{dim}\left(X_{s}\right)$ and $L_{s}^{\otimes_{i}}$ is spanned by its global sections for some $i>0$ then $s \in W$.

More generally if $d=K\left(X_{s}, L_{s}\right) \leqslant \operatorname{dim}\left(X_{s}\right)$ and for some $i>0$ the map $f_{i, s}: X_{s} \rightarrow \mathbf{P}^{N}$ given by $L_{s}^{\otimes_{i}}$ is everywhere defined, $\operatorname{dim}\left(f_{i, s}\left(X_{s}\right)\right)=d$, and $\operatorname{dim}\left(\operatorname{supp} R^{1} f_{i s^{*}}(0)\right)<d$ then $s \in W$. Thus for pairs $\left(X_{s}, L_{s}\right)$ satisfying the preceding hypotheses, the $L$-dimension can only go up under deformation.

\section{REFERENCES}

1. S. Iitaka, Deformations of compact complex surfaces. II, J. Math. Soc. Japan 22 (1970), 247-261. MR 41 \#6252.

2. - On D-dimensions of algebraic varieties, J. Math. Soc. Japan 23 (1971), 356-373. MR 44 \#2749.

3. D. Mumford, The canonical ring of an algebraic surface, Ann. of Math. (2) 76 (1962), 612-615.

4. K. Ueno, On Kodaira dimensions of certain algebraic varieties, Proc. Japan Acad. 47 (1971), 157-159. MR 45 \#1911.

DEPARTMENT OF MATHEMATICS, BRANDEIS UNIVERSITY, WALTHAM, MASSACHUSETTS 02154 